\title{
Equidistribution in non-archimedean parameter curves towards the activity measures
}

\author{
By Reimi Irokawa*) and Yûsuke OKUYAmA*) \\ (Communicated by Kenji FukAyA, M.J.A., Sept. 13, 2021)
}

\begin{abstract}
For every pair of an analytic family $f=f_{t}$ of endomorphisms of degree $>1$ of the Berkovich projective line $\mathbb{P}^{1, \text { an }}$ over an algebraically closed and complete non-trivially valued field $K$ and an analytically marked point $a=a(t)$ in $\mathbb{P}^{1, \text { an }}$ both parametrized by a domain $V$ in the Berkovich analytification of a smooth projective algebraic curve $C / K$, we establish the equidistribution of the averaged pullbacks of any value in $\mathbb{P}^{1, \text { an }}$ but a subset of logarithmic capacity 0 under the sequence of the morphisms $a_{n}=a_{n}(t)=f_{t}^{n}(a(t)): V \rightarrow \mathbb{P}^{1, \text { an }}$, towards the activity measure $\mu_{(f, a)}$ on $V$ associated with $f$ and $a$.
\end{abstract}

Key words: Analytic family of morphisms; analitically marked point; activity measure; potential theory on Berkovich curves; equidistribution; Varilon exceptional set.

1. Introduction. Let $K$ be an algebraically closed field that is complete with respect to a nontrivial and non-archimedean absolute value. Let $V$ be a (topological) domain in the analytification (Berkovich [3]) of a smooth projective algebraic curve $C / K$. To the pair $(f, a)$, where $f$ is an analytic family

$$
f_{t}(z)=f(t, z): V \times_{K} \mathbb{P}^{1, \text { an }} \rightarrow \mathbb{P}^{1, \text { an }}=\mathbb{P}_{K}^{1, \text { an }}
$$

of endomorphisms of $\mathbb{P}^{1, \text { an }}$ of degree $d>1$ analytically parametrized by $V$ and $a: V \rightarrow \mathbb{P}^{1, \text { an }}$ is a marked point in $\mathbb{P}^{1, \text { an }}$ analytically parametrized by $V$ (i.e., an analytic map from $V$ to $\left.\mathbb{P}^{1, \text { an }}\right)$, the activity measure

$$
\mu_{(f, a)}:=\lim _{n \rightarrow \infty} \frac{\left(a_{n}\right)^{*} \delta_{\zeta_{0,1}}}{d^{n}} \quad \text { weakly on } V
$$

is associated ([6]), where for every $n \in \mathbb{N}$,

$$
a_{n}(t):=f_{t}^{n}(a(t)), \quad t \in V
$$

is a marked point in $\mathbb{P}^{1, \text { an }}$ analytically parametrized by $V$, and $\zeta_{0,1}$ is the Gauss (or canonical) point in $\mathbb{P}^{1, \text { an }}$ and $\delta_{\zeta}$ is the Dirac measure on $\mathbb{P}^{1, \text { an }}$ at a point $\zeta \in \mathbb{P}^{1, \text { an }}$. The activity measures of especially marked critical points play a key role in pluripotential theoretic studies (since [4]) of bifurcation

2010 Mathematics Subject Classification. Primary 37P50; Secondary 37P45.

*) Graduate School of Science, Tokyo Institute of Technology, 2-12-1 Ookayama, Meguro-ku, Tokyo 152-8551, Japan. **) Division of Mathematics, Kyoto Institute of Technology, Matsugasaki, Sakyo-ku, Kyoto 606-8585, Japan. and (un)stability in complex dynamics (foundationally [7-9]). A non-archimedean version of bifurcation and (un)stability including a non-archimedean $\lambda$-lemma has been studied by Thomas Silverman [11].

Our principal result is the following, which has been expected in [6].

Theorem 1. Let $K$ be an algebraically closed field that is complete with respect to a nontrivial and non-archimedean absolute value, and $V$ be a domain in the (Berkovich) analytification of a smooth projective algebraic curve $C / K$. Let $f$ : $V \times_{K} \mathbb{P}^{1, \text { an }} \rightarrow \mathbb{P}^{1, \text { an }}$ be a family of endomorphisms of $\mathbb{P}^{1, \text { an }}$ of degree $d>1$ and $a: V \rightarrow \mathbb{P}^{1, \text { an }}$ be a marked point in $\mathbb{P}^{1, \text { an }}$, both analytically parametrized by $V$. Then for every $\zeta \in \mathbb{P}^{1, \text { an }}$ but a subset of logarithmic capacity 0 ,

$$
\lim _{n \rightarrow \infty} \frac{\left(a_{n}\right)^{*} \delta_{\zeta}}{d^{n}}=\mu_{(f, a)} \quad \text { weakly on } V .
$$

Indeed, we would establish the following, which is motivated by Nevanlinna theory.

Theorem 2. Under the same assumption in Theorem 1, the Valiron exceptional set

$$
\begin{aligned}
& E_{V}(f, a) \\
& \quad:=\left\{\zeta \in \mathbb{P}^{1, \text { an }} \mid \begin{array}{l}
\text { there is } x \in V \backslash V(K) \text { such that } \\
\log \left\|a_{n}(x), \zeta\right\| \neq o\left(d^{n}\right) \text { as } n \rightarrow \infty
\end{array}\right\} \\
& \text { associated with the pair }(f, a) \text { is of logarithmic } \\
& \text { capacity } 0 \text { in } \mathbb{P}^{1, \text { an }} \text {. } \\
& \quad \text { For the definition of the generalized Hsia kernel } \\
& \|\zeta, \xi\| \text { on } \mathbb{P}^{1, \text { an }} \text { with respect to } \zeta_{0,1} \text { and that of a }
\end{aligned}
$$


subset of $\mathbb{P}^{1, \text { an }}$ of logarithmic capacity 0 , see Section 2 below. A standard argument from potential theory on Berkovich curves (using a functoriality and a continuity of the Laplacian $\mathrm{dd}^{c}$ on Berkovich curves (see Thuillier [13, §3.2, §3.3])) shows that for every $\zeta \in \mathbb{P}^{1, \text { an }} \backslash E_{V}(f, a)$,

$$
\lim _{n \rightarrow \infty} \frac{\left(a_{n}\right)^{*}\left(\delta_{\zeta_{0,1}}-\delta_{\zeta}\right)}{d^{n}}=0 \quad \text { weakly on } V .
$$

Hence Theorem 2 together with (1.1) yields Theorem 1 .

Organization of this paper. In Section 2, we gather some background materials and notations from potential theory on the Berkovich projective line and curves. In Section 3, we show Theorem 2 when $V$ is separable. In Section 4, we show Theorem 2 in full generality.

2. Background. The Berkovich projective line $\mathbb{P}^{1, \text { an }}=\mathbb{P}_{K}^{1 \text {,an }}$ is a compact augmentation of $\mathbb{P}^{1}=\mathbb{P}_{K}^{1}$, and a typical point in $\mathbb{P}^{1, \text { an }}$ is written as $\zeta_{a, r}$ when it is represented by a $K$-closed disk $B(a, r):=\{z \in K:|z-a| \leq r\}$ in $K$ for some $a \in K$ and $r \geq 0 ; K=\mathbb{P}^{1} \backslash\{\infty\}$ is identified with the set of all points in $\mathbb{P}^{1, \text { an }}$ written as $\zeta_{a, 0}$ for some $a \in K$. The point $\zeta_{0,1}$ is called the Gauss (or canonical) point in $\mathbb{P}^{1, \text { an }}$. The chordal distance $\|z, w\|$ on $\mathbb{P}^{1}$ normalized as $\|0, \infty\|=1$ extends to a unique upper semicontinuous and separately continuous function on $\mathbb{P}^{1, \text { an }} \times \mathbb{P}^{1, \text { an }}$, which is still denoted by $\|\zeta, \xi\|$ and is called the generalized Hsia kernel function on $\mathbb{P}^{1, \text { an }}$ with respect to $\zeta_{0,1}$; in particular, $\|\zeta, \zeta\|=0$ if and only if $\zeta \in \mathbb{P}^{1}$. For every point $\zeta_{0} \in \mathbb{P}^{1 \text {,an }}$, the function $\log \left\|\cdot, \zeta_{0}\right\|$ on $\mathbb{P}^{1 \text {,an }}$ is locally constant except for the closed interval $\left[\zeta_{0}, \zeta_{0,1}\right]$ between $\zeta_{0}$ and $\zeta_{0,1}$ in $\mathbb{P}^{1, \text { an }}$. An analytic map $h$ from a domain $D$ in the Berkovich analytification of a smooth projective curve $C / K$ to $\mathbb{P}^{1 \text {,an }}$ induces a canonical pullback operator $h^{*}$ from the space of Radon measures on $\mathbb{P}^{1 \text {,an }}$ to that of Radon measures on $D$ (for more details on $\mathbb{P}^{1 \text {,an }}$, see [5, Chapter 4], [5, §3.4]).

We adopt the following sign convention on the Laplacian $\mathrm{dd}^{c}$ on Berkovich curves; for every $\zeta \in \mathbb{P}^{1, \text { an }}$,

$$
-\mathrm{dd}^{c} \log \|\cdot \zeta\|=\delta_{\zeta}-\delta_{\zeta_{0,1}} \quad \text { on } \mathbb{P}^{1, \text { an }}
$$

We call a function

$$
u_{\zeta_{0,1}, \rho}(\cdot):=-\int_{\mathbb{P}^{1, \mathrm{an}}} \log \|\cdot, \zeta\| \rho(\zeta) \in[0,+\infty]
$$

$$
\text { (so that } \operatorname{dd}^{c} u_{\zeta_{0,1}, \rho}=\rho-\rho\left(\mathbb{P}^{1, \text { an }}\right) \zeta_{0,1} \text { on } \mathbb{P}^{1, \text { an }} \text { ) }
$$

on $\mathbb{P}^{1, \text { an }}$ the logarithmic potential function of a positive Radon measure $\rho$ on $\mathbb{P}^{1, \text { an }}$ with respect to $\zeta_{0,1}$. The logarithmic capacity (with respect to $\zeta_{0,1}$ ) of a subset $E$ of $\mathbb{P}^{1, \text { an }} \backslash\left\{\zeta_{0,1}\right\}$ is

$$
\operatorname{Cap} E:=\exp \left(-\inf _{\rho} \int_{\mathbb{P}^{1, \text { an }}} u_{\zeta_{0,1}, \rho}(\xi) \rho(\xi)\right) \in[0,1],
$$

where $\rho$ ranges over all probability Radon measures on $\mathbb{P}^{1, \text { an }}$ supported by $E$, and we say $E$ is of logarithmic capacity 0 (with respect to $\zeta_{0,1}$ ) if Cap $E=0$. If $E$ is not of logarithmic capacity 0 , then there is a compact subset of $E$ which is not of logarithmic capacity 0 . If a compact subset $\mathcal{C}$ of $\mathbb{P}^{1 \text {,an }} \backslash\left\{\zeta_{0,1}\right\}$ is not of logarithmic capacity 0 , then a unique equilibrium mass distribution $\rho_{\mathcal{C}}$ on $\mathcal{C}$ with respect to $\zeta_{0,1}$ (i.e., a probability Radon measure $\rho$ on $\mathbb{P}^{1, \text { an }}$ supported by $\mathcal{C}$ and satisfying $\operatorname{Cap} \mathcal{C}=$ $\left.\exp \left(-\int_{\mathbb{P}^{1, \text { an }}} u_{\zeta_{0,1}, \rho}(\xi) \rho(\xi)\right)\right)$ exists. For more details on the logarithmic capacity theory on $\mathbb{P}^{1, \text { an }}$, see [2, Chapter 6].

\section{Proof of Theorem 2: separable domain} case. Let $V$ be a domain in the (Berkovich) analytification of a smooth projective algebraic curve $C / K$. Let $f: V \times_{K} \mathbb{P}^{1, \text { an }} \rightarrow \mathbb{P}^{1 \text {,an }}$ be a family of endomorphisms of $\mathbb{P}^{1, \text { an }}$ of degree $d>1$ and $a$ : $V \rightarrow \mathbb{P}^{1, \text { an }}$ be a marked point in $\mathbb{P}^{1, \text { an }}$, both analytically parametrized by $V$. Recall the definition (1.2) of the marked point $a_{n}$ for each $n \in \mathbb{N}$.

For every $x \in V \backslash V(K)$, the subset

(3.1) $\mathcal{E}_{x}=\mathcal{E}_{x}(f, a)$

$$
:=\bigcup_{j \in \mathbb{N}} \bigcap_{N \in \mathbb{N}} \bigcup_{n \geq N}\left\{\zeta \in \mathbb{P}^{1, \text { an }} \mid\left\|a_{n}(x), \zeta\right\|<e^{\frac{-d^{n}}{j}}\right\}
$$

of $\mathbb{P}^{1, \text { an }}$ is the countable union of subsets of $\mathbb{P}^{1}$ of finite Hyllengren measures for the increasing sequence $\left(d^{n}\right)_{n}$ (cf. [12]), so in particular is of logarithmic capacity 0 (for a more direct argument, see e.g. [10, Proof of Lemma 2.1]).

Suppose now that $V$ is separable, that is, $V \backslash$ $V(K)$ contains a countable dense subset $S$ (as in e.g. the case that $K=\mathbb{C}_{p}$ ). Then the subset

$$
\mathcal{E}_{S}:=\bigcup_{x \in S} \mathcal{E}_{x}
$$

of $\mathbb{P}^{1 \text {,an }}$ is of logarithmic capacity 0 . We claim that $E_{V}(f, a) \subset \mathcal{E}_{S} ;$ for, otherwise, there are $\zeta_{0} \in$ $\mathbb{P}^{1, \text { an }} \backslash \mathcal{E}_{S}, x_{0} \in V \backslash V(K)$, and a sequence $\left(n_{j}\right)$ in $\mathbb{N}$ tending to $\infty$ such that 


$$
\lim _{j \rightarrow \infty} \frac{\log \left\|a_{n_{j}}\left(x_{0}\right), \zeta_{0}\right\|}{d^{n_{j}}}<0 .
$$

From [6], we can pick a connected open affinoid neighborhood $U$ of $x_{0}$ in $V$ so small that for a nondegenerate homogeneous polynomial lift $F: U \times_{K}$ $\mathbb{A}^{2 \text {,an }} \rightarrow \mathbb{A}^{2 \text {,an }}=\mathbb{A}_{K}^{2 \text {,an }}$ of $f: U \times_{K} \mathbb{P}^{1, \text { an }} \rightarrow \mathbb{P}^{1, \text { an }}$ and an analytic lift $A: U \rightarrow \mathbb{A}^{2 \text {,an }} \backslash\left\{0_{\mathbb{A}_{K}^{2}}\right\}$ of $a: U \rightarrow$ $\mathbb{P}^{1, \text { an }}$, writing $F^{n}=\left(F_{0}^{(n)}, F_{1}^{(n)}\right)$ for each $n \in \mathbb{N}$, the function

$$
h^{(n)}(t):=\frac{\log \max \left\{\left|F_{0}^{(n)}(t, A(t))\right|,\left|F_{1}^{(n)}(t, A(t))\right|\right\}}{d^{n}}
$$

on $U(K)$ extends continuously and subharmonically to $U$ so that $-\mathrm{dd}^{c} h^{(n)}=a_{n}^{*} \delta_{\zeta_{0,1}} / d^{n}$ on $U$, and the uniform limit $h_{(f, a)}=\lim _{n \rightarrow \infty} h^{(n)}$ on $U$ exists and is a continuous and subharmonic function on $U$. Then for every $j \in \mathbb{N}$, the function

$$
\frac{\log \left\|a_{n_{j}}(\cdot), \zeta_{0}\right\|}{d^{n_{j}}}+h^{\left(n_{j}\right)} \quad \text { on } U
$$

is subharmonic (using a functoriality of $\mathrm{dd}^{c}$ ), and shrinking $U$ if necessary, the family of those subharmonic functions on $U$ is also uniformly bounded from above on $U$. Then using a nonarchimedean version of Hörmander's version of Hartogs's lemma (see [2, Proposition 8.54]), either

$$
\lim _{j \rightarrow \infty} \frac{\log \left\|a_{n_{j}}(\cdot), \zeta_{0}\right\|}{d^{n_{j}}}=-\infty
$$

uniformly on any compact subset of $U$ or, taking a subsequence of $\left(n_{j}\right)$ if necessary, there is an upper semicontinuous function $\phi$ on $U$ such that

$$
\phi=\lim _{j \rightarrow \infty} \frac{\log \left\|a_{n_{j}}(\cdot), \zeta_{0}\right\|}{d^{n_{j}}} \quad \text { on } U \backslash U(K)
$$

(so $\{\phi<0\}$ in $U$ is open and contains $x_{0}$ ). In the former case, we must have $\zeta_{0} \in \mathcal{E}_{S}$ since $U \cap S \neq \emptyset$. In the latter case, we must still have $\zeta_{0} \in \mathcal{E}_{S}$ since $\{\phi<0\} \cap S \neq \emptyset$. In any case, this is a contradiction.

Hence $E_{V}(f, a) \subset \mathcal{E}_{S}$, which completes the proof of Theorem 2 in this case.

4. Proof of Theorem 2: general case. Let $V$ be a domain in the (Berkovich) analytification $C^{\text {an }}$ of a smooth projective algebraic curve $C / K$. Let $f: V \times_{K} \mathbb{P}^{1, \text { an }} \rightarrow \mathbb{P}^{1, \text { an }}$ be a family of endomorphisms of $\mathbb{P}^{1, \text { an }}$ of degree $d>1$ and $a: V \rightarrow \mathbb{P}^{1 \text {,an }}$ be a marked point in $\mathbb{P}^{1, \text { an }}$, both analytically parame- trized by $V$. Recall the definition (1.2) of the marked point $a_{n}$ for each $n \in \mathbb{N}$.

Lemma 4.1. For any probability Radon measure $\rho$ on $\mathbb{P}^{1, \text { an }}$, we have $\operatorname{Cap}\left(E_{V}(f, a) \cap\right.$ $(\operatorname{supp} \rho))=0$. $\mathbb{P}^{1, \text { an }}$.

Proof. Pick a probability Radon measure $\rho$ on

Recall the profinite graph (more precisely, the inverse limit of skeletons) structure of the Berkovich curve $C^{\text {an }}$ and the (extended) skeletal metric on $C^{\text {an }} \backslash C(K)$ (see $\left.[1, \S 5]\right)$. Here, a skeleton $\Sigma$ of $C^{\text {an }}$ is a finite subgraph in $C^{\text {an }}$ so that all connected components of $C^{\text {an }} \backslash \Sigma$ are open balls in $C^{\text {an }}$ (see $[1, \S 3])$. In particular, by the connectedness of $V$ and the compactness of the topological boundary $\partial V$ of $V$ in $C^{\text {an }}$, there is a sequence $\left(U_{j}\right)_{j}$ of relatively compact subdomains in $V$ increasing to $V$ such that for any $j \in \mathbb{N}, U_{j}$ is a connected component of the complement in $C^{\text {an }}$ of a finite subset of $C^{\text {an }}$.

Let $\Gamma_{0}$ be the union of all paths in $V$ joining distinct two points in $\partial V$. Then for every $j \in \mathbb{N}$, $\Gamma_{0}^{(j)}:=\Gamma_{0} \cap U_{j}$ is an at most finitely branched and connected subgraph in $U_{j}$. For any $j, n \in \mathbb{N}$, the signed measure $\left(a_{n}^{*}\left(\rho-\delta_{\zeta_{0,1}}\right)\right) \mid \overline{U_{j}}$ is finite, so for every $j \in \mathbb{N}$, by the argument in $[2$, Proof of Lemma $5.7]$, there is an increasing sequence $\left(\Gamma_{k}^{(j)}\right)_{k \in \mathbb{N}}$ of finite and connected subgraphs in $U_{j}$ such that $\bigcup_{n \in \mathbb{N}}\left(\operatorname{supp}\left(a_{n}^{*}\left(\rho-\delta_{\zeta_{0,1}}\right)\right)\right) \cap U_{j}$ is contained in the closure of $\bigcup_{k \in \mathbb{N}} \Gamma_{k}^{(j)}$ in $V$. Noting that for $j \gg 1, U_{j}$ contains the union $L$ of all (finitely many nontrivial) loops in $V$, for every $j \gg 1$ and every $k \in \mathbb{N} \cup\{0\}$, we replace $\Gamma_{k}^{(j)}$ with an at most finitely branched and connected subgraph in $U_{j}$ containing $\Gamma_{k}^{(j)} \cup L$. Then letting

$$
X_{\rho}=X_{\rho}^{(f, a)}:=\overline{\bigcup_{j \in \mathbb{N}}\left(\bigcup_{k \in \mathbb{N} \cup\{0\}} \Gamma_{k}^{(j)}\right)} \text { in } V,
$$

we have

$$
\bigcup_{\zeta \in \operatorname{supp} \rho}\left(\bigcup_{n \in \mathbb{N}} a_{n}^{-1}\left(\left[\zeta, \zeta_{0,1}\right]\right)\right) \subset X_{\rho},
$$

and there is a countable dense subset $\mathcal{Y}_{\rho}$ of $X_{\rho}$. Set

$$
\mathcal{E}_{\mathcal{Y}_{\rho}}:=\bigcup_{y \in \mathcal{Y}_{\rho}} \mathcal{E}_{y}
$$

which is still of logarithmic capacity 0 in $\mathbb{P}^{1, \text { an }}$. Here for each $y \in V \backslash V(K)$, the subset $\mathcal{E}_{y}$ of $\mathbb{P}^{1 \text {,an }}$ (indeed of $\left.\mathbb{P}^{1}\right)$ is defined as in $(3.1)$.

We claim that $E_{V}(f, a) \cap(\operatorname{supp} \rho) \subset \mathcal{E}_{\mathcal{Y}_{\rho}}$; for, otherwise, there are $\zeta_{0} \in\left((\operatorname{supp} \rho) \cap E_{V}(f, a)\right) \backslash \mathcal{E}_{\mathcal{Y}_{\rho}}$, 
$x_{0} \in V \backslash V(K)$, and a sequence $\left(n_{j}\right)$ in $\mathbb{N}$ tending to $\infty$ such that

$$
\lim _{j \rightarrow \infty} \frac{\log \left\|a_{n_{j}}\left(x_{0}\right), \zeta_{0}\right\|}{d^{n_{j}}}<0 .
$$

Suppose that $x_{0} \in V \backslash X_{\rho}$, and let $U$ be the connected component of $V \backslash X_{\rho}$ containing $x_{0}$. Then for every $j \in \mathbb{N}$, the continuous function $\left(\log \left\|a_{n_{j}}(\cdot), \zeta_{0}\right\|\right) / d^{n_{j}}$ on $V$ is constant on $U$ (for, otherwise, since the generalized Hsia kernel $\left\|\cdot, \zeta_{0}\right\|$ is locally constant on $\mathbb{P}^{1, \text { an }} \backslash\left[\zeta_{0}, \zeta_{0,1}\right]$, by $\zeta_{0} \in \operatorname{supp} \rho$ and (4.1), we must have $\emptyset \neq U \cap a_{n_{j}}^{-1}\left(\left[\zeta_{0}, \zeta_{0,1}\right]\right) \subset X_{\rho}$, which is impossible). This with (4.2) yields the convergence

$$
\begin{aligned}
\lim _{j \rightarrow \infty} & \frac{\log \left\|a_{n_{j}}(\cdot), \zeta_{0}\right\|}{d^{n_{j}}} \\
& \equiv \lim _{j \rightarrow \infty} \frac{\log \left\|a_{n_{j}}\left(x_{0}\right), \zeta_{0}\right\|}{d^{n_{j}}}<0 \text { on } \bar{U} .
\end{aligned}
$$

Hence, since $\emptyset \neq \partial U \subset X_{\rho} \backslash V(K)$, where $\partial U$ is the topological boundary of $U$ in $C^{\text {an }}$, we can replace the original $x_{0}$ so that $x_{0} \in X_{\rho}$ (without changing the $\left.\zeta_{0}\right)$. Then we are done by an argument by contradiction similar to that in the separable case in Section 3 which involves a non-archimedean version of Hörmander's version of Hartogs's lemma. Hence the claim holds.

Once this claim is at our disposal, we are done by $\operatorname{Cap}\left(\mathcal{E}_{\mathcal{Y}_{\rho}}\right)=0$ and the monotonicity of the capacity function Cap.

If $E_{V}(f, a)$ is not of logarithmic capacity 0 , then there must exist a probability Radon measure $\rho$ on $\mathbb{P}^{1, \text { an }}$ supported by $E_{V}(f, a)$ and satisfying $\int_{\mathbb{P}^{1, \text { an }}} u_{\zeta_{0,1}, \rho}(\xi) \rho(\xi)<\infty$. By Lemma 4.1 , this is impossible (see [2, Lemma 6.16]). Now the proof of Theorem 2 is complete.

Acknowledgements. The first author was partially supported by JSPS Grant-in-Aid for JSPS Fellows, 20J14309. The second author was partially supported by JSPS Grant-in-Aid for Scientific Research (C), 19K03541 and (B), $19 \mathrm{H} 01798$.

\section{References}

[ 1 ] M. Baker, S. Payne and J. Rabinoff, On the structure of non-Archimedean analytic curves, in Tropical and non-Archimedean geometry, Contemp. Math., 605, Centre Rech. Math. Proc., Amer. Math. Soc., Providence, RI, 2013, pp. 93-121.

[ 2 ] M. Baker and R. Rumely, Potential theory and dynamics on the Berkovich projective line, Mathematical Surveys and Monographs, 159, American Mathematical Society, Providence, RI, 2010.

[ 3 ] V. G. Berkovich, Spectral theory and analytic geometry over non-Archimedean fields, Mathematical Surveys and Monographs, 33, American Mathematical Society, Providence, RI, 1990.

[ 4 ] L. DeMarco, Dynamics of rational maps: Lyapunov exponents, bifurcations, and capacity, Math. Ann. 326 (2003), no. 1, 43-73.

[ 5 ] C. Favre and J. Rivera-Letelier, Équidistribution quantitative des points de petite hauteur sur la droite projective, Math. Ann. 335 (2006), no. 2, $311-361$

[ 6 ] R. Irokawa, Activity measures of dynamical systems over non-archimedean fields, arXiv: 1901.01075.

[ 7 ] M. Yu. Lyubich, Some typical properties of the dynamics of rational maps, Russian Math. Surveys 38 (1983), no. 5, 154-155.

[ 8 ] R. Mañé, P. Sad and D. Sullivan, On the dynamics of rational maps, Ann. Sci. École Norm. Sup. (4) 16 (1983), no. 2, 193-217.

[ 9 ] C. T. McMullen and D. P. Sullivan, Quasiconformal homeomorphisms and dynamics. III. The Teichmüller space of a holomorphic dynamical system, Adv. Math. 135 (1998), no. 2, 351-395.

[10 ] Y. Okuyama, Fekete configuration, quantitative equidistribution and wandering critical orbits in non-archimedean dynamics, Math. Z. 273 (2013), no. $3-4,811-837$.

[11] T. Silverman, A non-archimedean $\lambda$-lemma, arXiv:1712.01372.

[12] M. Sodin, Value distribution of sequences of rational functions, in Entire and subharmonic functions, Adv. Soviet Math., 11, Amer. Math. Soc., Providence, RI, 1992, pp. 7-20.

[13 ] A. Thuillier, Théorie du potentiel sur les courbes en géomérie analytique non archimédienne. Applications à la théorie d'Arakelov, (2005). (Ph.D thesis, Université Rennes 1). 